\title{
INVARIANT SETS OF EQUATIONS IN RIEMANN SPACE*
}

\author{
BY \\ PHILIP FRANKLIN
}

\section{INTRODUCTION}

It is assumed in the theory of relativity that physical quantities are represented by expressions derived from tensor components, and that the laws of nature may be expressed as equations stating the equality of two tensors. This assumption is made so as to satisfy the requirement that physical laws must be expressible in a form independent of the particular coördinates used. If we start with this latter assumption, we are tempted to require merely that the equations expressing a law of nature be invariant, as a set, under transformations of coordinates, and the question arises as to the relation of equations of this type, referred to in the sequel as an invariant set of equations, to the tensor equations usually assumed. The requirement of invariance implies that there is a law of transformation for the equations in terms of the transformation of coordinates, which will be given if the equations involve merely tensor components and the coördinates. If these quantities enter into the equations in a sufficiently simple manner (which, however, is as general as is required in most of the equations of physics), we may completely answer the question raised above by the theorem

An invariant set of equations whose members are formed from the components of one or more tensors and point functions by addition, multiplication, and differentiation with respect to the coördinates is equivalent to a set of tensor equations.

Here, as throughout this paper, the tensors relate to a Riemann $n$-space.

We apply this theorem to the classification of invariant equations involving the derivatives of the fundamental quadratic tensor $\left(g_{i j}\right)$ and show that

An invariant set of equations involving the derivatives of the $g_{i j}$, the second derivatives appearing linearly, and no derivative higher than the second occurring, is equivalent to one of five standard tensor equations.

A theorem nearly equivalent to this for 4-space was given by G.D. Birkhoff.t Besides holding for $n$-space, our discussion is free trom the assumptions that the equations are homogeneous, which rules out Einstein's "cosmological equation", and that the coordinates have a certain reality character.

* Presented to the Society, April 28, 1923.

† Relativity and Modern Physics, Harvard University Press, 1923, pp. 211-220. 
The above theorems constitute the chief results of this paper. We proceed to the proofs.

\section{EqUATIONS LINEAR IN A SINGLE TENSOR}

We shall begin with the special case of equations involving the components of a single tensor, and these linearly, and shall prove that such an invariant set is equivalent to a set of tensor equations, the left members of these equations being linear combinations of the single tensor given, and tensors easily derived from it, with scalar coefficients. To fix the ideas, we shall give the details in full only for a tensor of the fourth order; the methods are, however, general.

If we consider our equations at a single point, the coefficients of the tensor components, which in general are point functions, become constants. If we further introduce normal (orthogonal geodesic) coordinates at this point, we have there

$$
g_{i j}=\delta_{i j}, \quad \partial g_{i j} / \partial x_{k}=0 \text {, }
$$

and the transformations of coordinates which change one such system into another are those belonging to the orthogonal group. Since our equations remain invariant under all coordinate transformations, they retain their form (as a set) for any linear orthogonal transformation. This leads to the

LEMMA. An invariant set of equations linear in a single tensor is equivalent to a set of equations each of which has the property that any subscript appears an odd number of times in every one of its terms, or an even number, perhaps zero.

We prove this by noting that when we perform the transformation

$$
x_{1}^{\prime}=-x_{1}, \quad x_{i}^{\prime}=x_{i} \quad(i \neq 1),
$$

which corresponds to a reflection in the 1-axis, any term containing the subscript 1 an odd number of times has its sign changed, while any one containing it an even number of times is unaffected. Hence if we apply the reflection (2) to any one of our equations, we obtain a new equation which is a consequence of our given set, from the invariant character, and on being added to and subtracted from the original equation gives rise to two equations of the type required by the lemma for the subscript 1 . By repeating the process for the remaining subscripts, we reach the desired result.

We turn now to the set of equations linear in the components of $P_{a b c d}$, the tensor of the fourth order. Suppose first that some one of our equations contains a term with four distinct subscripts, say 1234 (all the subscripts, if in four-space, a particular group if in $n$-space $(n>4)$; if in a space of less than four dimensions, there are no such terms, and our argument proceeds at once 
to the place below where terms with subscripts not all distinct are aiscussed). By applying the lemma, we may then obtain an equation in which every term contains these four distinct subscripts an odd number of times, and therefore each once. Let this equation be

$$
A P_{1884}+B P_{1842}+\cdots=0 .
$$

If we define the new tensor

$$
Q_{a b c d}=A P_{a b c d}+B P_{a c d b}+\cdots,
$$

we see that (3) gives

$$
Q_{1834}=0 .
$$

Since our set of equations is invariant, (3) and hence (5) holds in all systems of corrdinates. In particular, since we may transform the coorrdinates by a permutation of the variables, we see that all the components of $Q_{a b c d}$ with four distinct subscripts vanish.

We shall obtain the tensor equation which follows from our set by determining constants $A, B$, etc. for which the equation

$$
\begin{aligned}
Q_{a b c d}= & \delta_{a b}\left(A Q_{i i c d}+B Q_{i i d c}+C Q_{i c d i}+D Q_{i d c i}+E Q_{i c i d}+F Q_{i d i c}\right. \\
& \left.+G Q_{c d i i}+H Q_{d c i i}+I Q_{c i i d}+J Q_{d i i c}+K Q_{c i d i}+L Q_{d i c i}\right) \\
& +\delta_{a c}\left(A^{\prime} Q_{i i b d}+B^{\prime} Q_{i i d b}+\cdots\right)+\cdots \\
& +\delta_{a b} \delta_{c d}\left(U Q_{i i j j}+V Q_{i j i}+W Q_{i j i j}\right)+\cdots \\
& +\delta_{a c} \delta_{b d}\left(U^{\prime} Q_{i i j j}+V^{\prime} Q_{i j i}+W^{\prime} Q_{i j i j}\right)+\cdots
\end{aligned}
$$

is satisfied.

This equation holds regardless of the values of the constants, provided all four subscripts are distinct, in virtue of (5) and the similar equations. To see what conditions must be satisfied when two subscripts become equal, note that the equations

$$
\begin{aligned}
& x_{1}^{\prime}=x_{1} \cos \theta-x_{2} \sin \theta, \\
& x_{2}^{\prime}=x_{1} \sin \theta+x_{2} \cos \theta, \quad x_{i}^{\prime}=x_{i} \quad(i \neq 1,2)
\end{aligned}
$$


define an admissible transformation, and therefore our set of equations will hold after this transformation is applied. On applying it to (5), we obtain an equation in $\tan \theta$ true for all values of $\theta$. Consequently the coefficients must vanish, giving the new equation

$$
Q_{1134}=Q_{2234}
$$

On applying the rotation similar to (7), but involving the 1 and 3 -axes, to equation (8), which likewise holds for all systems of coorrdinates, multiplying the right side by $\cos ^{2} \theta+\sin ^{2} \theta$ to make the equation homogeneous, equating the coefficients to zero, and using (5), (8) and the similar equations, we find

$$
Q_{1114}=Q_{1824}+Q_{2184}+Q_{8214}
$$

If we now set $a b c d=1123$ in (6), and make use of (8), (9) and the similar equations, we obtain

$$
\begin{aligned}
Q_{1123} & =Q_{1123}(n A+D+E+I+L)+Q_{2311}(D+F+n G+I+K) \\
& +Q_{1182}(n B+C+F+J+K)+Q_{3211}(C+E+n H+J+L) \\
& +Q_{1231}(B+n C+E+H+K)+Q_{2113}(A+E+G+n I+K) \\
& +Q_{1321}(A+n D+F+G+L)+Q_{3112}(B+F+H+n J+L) \\
& +Q_{1813}(A+C+n E+H+I)+Q_{2181}(B+C+G+I+n K) \\
& +Q_{1812}(B+D+n F+G+J)+Q_{3121}(A+D+H+J+n L) .
\end{aligned}
$$

The equations obtained by making this an identity in the $Q$ 's and equating coefficients have a determinant equal to $n^{8}(n-2)^{5}(n+4)(n+2)^{3}$ which is different from zero (since $n \geqq 4$, to make this part of the argument necessary). Hence the equations have a solution, and when they are solved and the result is substituted in (6) it will hold for $a b c d=1123$. It will also hold for any choice of the subscripts making the first two equal, and the remaining two distinct from these and from each other, as is evident from the equations determining the constants.

In an entirely analogous way, we may determine the values of $A^{\prime}, B^{\prime}, \ldots, L^{\prime}$ so that the equation (6) will hold when $a$ and $c$ are equal, $b$ and $d$ being distinct from these and from each other; and then $A^{\prime \prime}, \ldots, A^{\prime \prime \prime}, \ldots, A^{\mathrm{vi}}, \ldots$ so that the equation will hold when any pair of subscripts are equal, the remaining 
pair being distinct from these and each other. It then follows from (9) and the similar equations that (6) holds when three of the subscripts are equal, but different from the fourth.

We now define a tensor obtained from $Q_{a b c d}$ by subtracting the terms already determined:

$$
S_{a b c d}=Q_{a b c d}-\delta_{a b} A Q_{i i c d}-\cdots-\delta_{c d} L^{\mathrm{vi}} Q_{b i a i}
$$

From the method we used to determine the constants appearing in this equation, it follows that all the components of $S_{a b c d}$ containing one of the subscripts an odd number of times vanish. Furthermore, by contracting (11) we may express the contracted $S$ 's in terms of the contracted $Q$ 's. Consequently we may find $U, \nabla, W$ etc. to satisfy (6) provided we can find constants which satisfy

$$
S_{a b c d}=\delta_{a b} \delta_{c d}\left(X S_{i i j j}+Y S_{i j j i}+Z S_{i j i j}\right)+\cdots
$$

We already know that the equations

$$
\begin{aligned}
& S_{1284}=0, \\
& S_{1123}=0, \\
& S_{1114}=0
\end{aligned}
$$

hold, as well as the similar equations obtained from them by permuting the subscripts. On applying the rotation similar to (7) involving the 2-and 3-axes to (14), and noting that we have an identity in $\theta$, we find

$$
S_{1122}=S_{1133}
$$

and hence

$$
S_{1122}=S_{334}
$$

Again, by applying the rotation involving the 1- and 4-axes, and using the equations already set down, we find

$$
S_{1111}=S_{4114}+S_{1144}+S_{1414}
$$

and hence, by (16),

$$
S_{1111}=S_{2282}
$$


If we now set $a b c d=1122$ in (12) and utilize the relations just derived, we find that

$$
\begin{aligned}
S_{1128}=S_{1122} & \left.n^{2} X+n Y+n Z\right)+S_{1221}\left(n X+n^{2} Y+n Z\right) \\
& +S_{1212}\left(n X+n Y+n^{2} Z\right)
\end{aligned}
$$

The equations obtained by considering this equation an identity in $S$ have a determinant equal to $n^{3}(n+2)(n-1)^{2}$, different from zero $(n \geqq 2$, since we have two distinct subscripts). These equations may thus be solved for $X$, $Y$ and $Z$. Similarly we determine $X^{\prime}, Y^{\prime}, Z^{\prime}$ so that (12) holds for $S_{1221}$, and $X^{\prime \prime}, Y^{\prime \prime}, Z^{\prime \prime}$ so that it holds for $S_{1212}$. It then follows from the way these coefficients are determined that equation (12) holds for any component whose subscripts form two pairs of equal elements, all four not being the same. This last case, however, is covered by recalling (18) and noticing the form of (12).

Having thus determined the coefficients to satisfy (12), which is now true for all subscripts, we combine (12), (11) and the equations obtained by contracting (11) so as to get an equation of the form (6) which is true for all subscripts. On eliminating $Q_{a b c d}$ from this equation by means of (4) we obtain a tensor equation in $P_{a b c d}$ which is implied by our original set. If there are any other equations in the set in four distinct subscripts, which are not consequences of the tensor equation just obtained, we may apply the process again to get an additional tensor equation in $P_{a b c d}$. We may keep this up until the tensor equations obtained have as consequences all the equations of our set with four distinct subscripts. This will have to happen after at most 4 ! such tensor equations have been obtained, since from this number we could solve for all the components $P_{1234}, P_{1824}$ etc., and eliminate them from our original set.

When we have obtained these tensor equations, and removed from our original set of equations all those which are consequences of the tensor equations, we shall have a second set of equations. This residual set has the property that no member contains four distinct subscripts. If one of these equations contains one, and hence two indices (say 2,3) each an odd number of times, by applying the lemma we may obtain from it an equation in which every term contains these indices an odd number of times, and hence of the form

$$
m_{1123} P_{1123}+\cdots+m_{2283} P_{2823}+\cdots=0
$$

where the $m$ 's are mere numbers, coefficients of the components indicated by their subscripts. 
On applying the rotation similar to (7) involving the 1 - and 4-axes, to (21) and equating the coefficient of $\sin \theta$ to 0 , we find

$$
\left(m_{4428}-m_{1128}\right) P_{1483}+\text { terms in less than } 4 \text { subscripts }=0 .
$$

If the coefficient of $P_{1423}$ is not zero, the equation

$$
P_{1 \mathrm{183}}=0 \text {, }
$$

and hence (cf. (8))

$$
P_{\text {4188 }}=P_{1188}
$$

will be included in the consequences of our tensor equations derived from those involving four subscripts, and hence in view of (24) we can make

$$
m_{\mathrm{kss}}=m_{1128}
$$

in all cases.

If we next apply (7), involving the 1- and 2-axes, to (21), we find, as the condition for the coefficient of $\sin \theta \cos ^{2} \theta$ vanishing,

$$
\left(m_{2288}-m_{1183}-m_{1218}\right) P_{1283}+m_{248} P_{1488}+\cdots=0 .
$$

As this is of type (21), 1 and 3 being the odd subscripts, we obtain the relation, analogous to (25),

$$
m_{2283}-m_{1128}-m_{1218}=m_{248},
$$

or, in view of the relations similar to (25),

$$
m_{2283}=m_{1128}+m_{2118}+m_{1218} .
$$

In consequence of equations (25), (28) and similar equations, we may write (21) in the form

$$
m_{1128} P_{i i 28}+m_{1881} P_{i s s i}+\cdots=0
$$

which shows that the tensor

$$
T_{a b}=m_{118 s} P_{i j a b}+m_{18 s 1} P_{i a b i}+\cdots
$$


has all its components in two distinct subscripts zero, and by the method used above for $Q_{a b c d}$, we may show that $T_{a b}$ satisfies the tensor equation

$$
T_{a b}=\frac{1}{n} \delta_{a b} T_{i i}
$$

On eliminating $T_{a b}$ from (31) by means of (30) we obtain a tensor equation in $P_{a b c a}$. In the same way we obtain all possible tensor equations which result from the equations of our set with two distinct subscripts. We then reject all equations from the set which are consequences of any of the tensor equations so far obtained. The equations which remain, since they involve no subscript an odd number of times, must be of the form

$$
m_{1122} P_{1128}+\cdots+n_{1111} P_{1111}+\cdots=0 \text {. }
$$

On applying the rotation similar to (7) involving the 1- and 3-axes, we find, as the coefficient of $\sin \theta \cos ^{3} \theta$,

$$
\left(m_{1122}-m_{3328}\right) P_{1328}+\left(m_{1111}-m_{3113}-m_{1813}-m_{1138}\right) P_{1113}+\cdots=0 .
$$

By an argument similar to that used above to establish (25) we may show that the coefficients here either are zero, or can be made zero, giving

$$
\begin{aligned}
& m_{1128}=m_{3329}, \\
& m_{1111}=m_{3113}+m_{1313}+m_{1183} .
\end{aligned}
$$

Consequently we may write (32) in the form

$$
m_{1122} P_{i i j j}+m_{1821} P_{i j i}+\cdots+M=0,
$$

$M$ being a constant term. This is a tensor equation, and by obtaining all such equations from our set we will finally have a collection of tensor equations which is equivalent to our set, in the sense that it implies and is implied by the set. These equations are either of type (6), (31) using (30), or (36). All these may be included in a single form,

$$
A P_{a b c d} \cdots+B \delta_{a b} P_{i i c d} \cdots+C \delta_{a b} \delta_{c d} P_{i i j j} \cdots+\delta_{a b} \delta_{c d} D=0
$$


The coefficients in this equation are constants at the point under consideration, and the equation holds for normal coorrdinates at this point. As it is evidently equivalent to the equation in general coordinates

$$
A P_{a b c d} \cdots+B g_{a b} P_{i i c d} \cdots+C g_{a b} g_{c d} P_{i i j j} \cdots+g_{a b} g_{c d} D=0
$$

this may now be considered to hold at all points, if instead of regarding the coefficients as constants, we regard them as scalar point functions.

As the argument given above for tensors of the fourth order may evidently be extended to those of any order, the $m$ th, the process consisting in first deducing tensor equations from those in the set in $m$ distinct subscripts, then $m-2$, and so on, we may state as the conclusion of this section

THEOREM I. An invariant set of equations, linear in the components of a single tensor, is equivalent to a set of tensor equations, obtained by equating to zero linear combinations of the given tensor, those obtained from it by permuting the subscripts, and those obtained by contracting one or more times and multiplying by the fundamental quadratic tensor so as to bring the order to its original value. The coefficients in the linear relations are scalar point functions.

\section{EXTENSION TO THE GENERAL CASE}

The previous section merely dealt with equations linear in the components of a single tensor; it is, however, easy to extend the result there obtained to the case of equations formed from several tensors, differentiation and multiplication being admitted. This extension now concerns us.

Consider first the case where the equations are linear in the components of several tensors, not necessarily of the same order, so that each term involves only a component of one of the tensors. We confine our attention to one point, and introduce normal coordinates. The lemma of the preceding section evidently applies here, and by its use we may reduce our set to one in which each equation contains any one subscript an odd number of times, or an even number of times. Hence the tensors appearing in these equations must have orders differing by an even number, and they may all be brought up to the same order by introducing $\delta$ 's with equal numerical subscripts, which does not effect the invariant character of the set. Having done this, we may now select the equation of the set containing the greatest number of distinct subscripts, as we did in the preceding paragraph, and proceed to deduce tensor equations which follow from our set, exactly as before.

Next, if the equations of the set express the vanishing of expressions involving the components of several tensors to a degree higher than the first, being polynomials in these components, we have merely to regard the product 
of two components of the same, or of two different tensors, as a single component of a tensor whose order is the sum of the orders of the two used in forming it, to reduce this to the case just discussed.

Finally we consider the case where differentiation of the tensor components is permitted. We notice that we are dealing with normal coordinates in carrying out our reduction, and in these coordinates the first derivatives may be replaced by the corresponding covariant derivatives, while the higher derivatives may be replaced by polynomials in the higher covariant derivatives, and the derivatives of the Christoffel symbols in normal coordinates, evaluated at the origin. But these quantities are all tensors, ${ }^{*}$ and thus the set of equations implies a set holding in normal coordinates which may be treated by methods already given.

As an illustration of the reduction of the last three paragraphs, suppose one of our original equations were

$$
\left(W_{12}\right)^{3}=U_{12}+\left(\nabla_{12}\right)\left(\partial T_{1} / \partial x_{2}\right) .
$$

By applying the lemma, we should obtain, after replacing the derivative by a covariant derivative,

$$
\begin{gathered}
\left(V_{12}\right)\left(T_{1 / 2}\right)=0, \\
\left(W_{12}\right)^{3}-U_{12}=0,
\end{gathered}
$$

and on introducing the tensors defined by

we should obtain

$$
\begin{aligned}
Q_{a b c d} & =V_{a b} T_{c / d}, \\
Q_{a b c d e f}^{\prime} & =W_{a b} W_{c d} W_{e f}-\delta_{c e} \delta_{d f} U_{a b},
\end{aligned}
$$

$$
Q_{1812}=0,
$$

$$
Q_{121212}^{\prime}=0 \text {, }
$$

a form to which the result of the preceding section would apply.

We have thus proved the theorem stated in the introduction:

THEOREM II. An invariant set of equations, obtained by equating to zero expressions formed from one or more given tensors and point functions by

\footnotetext{
* For the expression of the derivatives of the Christoffel symbols at the origin of a system of normal coördinates in terms of the curvature tensor, see 0 . Veblen, Proceedings of the National Academy of Sciences, vol. 8 (1922), p. 196.
} 
addition, multiplication, and differentiation with respect to the coördinates, is equivalent to a set of tensor equations.

\section{EQUATIONS LINEAR IN THE SECOND DERIVATIVES OF THE FUNDAMENTAL QUADRATIC TENSOR}

In this section we shall consider the classification of invariant sets of equations, formed by equating to zero expressions involving the fundamental quadratic tensor, $g_{i j}$, its first and second derivatives, and these last linearly. Such equations are of interest, since the equations holding in space free of matter are of this form, so that on specializing our results to four-space they will throw light on the choice of equations for the relativistic theory of gravitation. The question is related to one concerning possible tensors of this type, which was previously taken up by the author.* The results of that paper, while related to those here obtained, neither follow from them nor lead to them.

As our invariant set of equations involve merely the $g_{i j}$, and their first and second derivatives, if we introduce normal coordinates, for which (1) holds, they will reduce to expressions in the second derivatives, and as these (in normal coördinates) are expressible in terms of the curvature tensor, we see that our equations involve this tensor only. Also, on account of the linearity requirement, they involve its components linearly, so that they are the type discussed in Section 2. By that section, we see that our equations must all be of the form

$$
\begin{gathered}
A R_{a b c d}+B R_{a d b c}+C g_{a b} K_{c d}+D g_{c d} R_{a b}+E g_{a c} R_{b d}+F g_{b d} R_{a c} \\
+H g_{a d} R_{b c}+I g_{b c} R_{a d}+J g_{a b} g_{c d} R+K g_{a c} g_{b d} R \\
+L g_{a d} g_{b c} R+M g_{a b} g_{c d}+N g_{a c} g_{b d}+P g_{a d} g_{b c}=0
\end{gathered}
$$

The terms in (38) omitted in this equation depend on the ones kept, owing to the symmetry relations of $\boldsymbol{R}_{a b c d}$, and we use the customary notation for the tensors obtained from it by contraction.

As we may be able to factor out the fundamental quadratic tensor from (43), in case every term contains a $g_{a b}$, say, - i. e., if all the coefficients were zero except $C, J, M$, - the factoring being accomplished by contracting with respect to $a$ and $b$, the equation may reduce to one of the second order, or to a scalar relation. We shall treat these simpler cases first. The scalar relation will evidently reduce to the form

$$
\boldsymbol{R}=\boldsymbol{M},
$$

$M$ being a scalar, perhaps zero.

*Philosophical Magazine, vol. 45 (1923), p. 998 ff. Cf. H.Weyl, Raum, Zeit, Materie, fourth edition, p. 287. 
If the equation reduces to one of the second order, it will be of the form

$$
C R_{c d}+J g_{c d} R+M g_{c d}=0 .
$$

On contracting this with respect to $c$ and $d$, we obtain

$$
C R+n J R+n M=0,
$$

$n$ being the dimensionality of the space. If $C$ is zero, this either gives an equation of type (44), having (45) as a consequence, or all the constants are zero and (45) is satisfied identically. If $C \neq 0$, on replacing $J R+M$ by $-C / n$ in (45), and dividing out $C$, we find

$$
R_{c d}-\frac{1}{n} g_{c d} R=0
$$

as the standard form for a tensor equation of the second order.

We may show that if, in (43), both $A$ and $B$ are zero, that equation is equivalent to one of type (44) or (47). For in that case, on contracting with respect to $a$ and $b$, we would obtain

$$
\begin{aligned}
(n C+E+F+ & H+I) R_{c d} \\
& +(D+n J+K+L) g_{c d} R+(n M+N+P) g_{c d}=0 .
\end{aligned}
$$

If the coefficient of $\boldsymbol{R}_{c d}$ in (48) is different from zero, it leads to an equation of type (47), by means of which we may eliminate all the $R$ 's with two subscripts from (43), the resulting equation easily reducing to a relation like (44). As these two equations of our earlier types would have (43) as a consequence, nothing new results from this case. If the coefficient of $R_{c d}$ in (48) were zero, we could contract (43) with respect to a second pair of subscripts and carry out the argument as before, unless all six coefficients were zero, in which case we would have $C=D=E=F=H=I=0$, and (43) would be essentially a scalar equation like (44).

When $A$ and $B$ are not both zero, by using the relation

$$
R_{a b c d}+R_{a d b c}+R_{a c d b}=0
$$

we can obtain a relation involving only one of these. For, since (43) is true for all coördinates, and hence in particular when we permute the subscripts, it gives, on interchanging $b$ and $c$,

$$
A R_{a c b d}+B R_{a d c b}+\cdots=0,
$$


or, using the symmetry properties of $R_{a b c d}$,

$$
-A R_{a c d b}-B R_{a d b c}+\cdots=0 .
$$

On subtracting this equation from (43), and using (49), we find

$$
(2 B-A) R_{a d b c}+\cdots=0 .
$$

If the coefficient is zero, we interchange the rôles of $A$ and $B$ in the argument, and as they are not both zero, we see that in all cases an equation of form (43) is obtained containing a single $R$ with four subscripts, with non-vanishing coefficient. Equation (52) may thus be solved for $R_{a d b c}$, and if we use this value in the left member of the identity

$$
R_{b a d c}-R_{b a c d}+R_{a b c d}-R_{a b d c}=4 R_{a b c d}
$$

which follows from the symmetry properties of $R_{a b c d}$, the resulting equation takes the form

$$
\begin{aligned}
& A R_{a b c d}+B\left(g_{a c} R_{b d}+g_{b d} R_{a c}-g_{a d} R_{b c}-g_{b c} R_{a d}\right) \\
& +C\left(g_{a c} g_{b d}-g_{a d} g_{b c}\right) R+D\left(g_{a c} g_{b d}-g_{a d} g_{b c}\right)=0 .
\end{aligned}
$$

Furthermore, (43) can not imply anything more than (54) unless perhaps equations of our earlier types, since we can eliminate all the terms with $R$ 's of four subscripts from (43) by using (54), coming · back to the case where $A$ and $B$ in (43) are both zero.

On contracting (54) with respect to $a$ and $d$, we find

$$
(A+(2-n) B) R_{b c}+(-B+(1-n) C) g_{b c} R+D(1-n) g_{b c}=0 \text {. }
$$

If the first coefficient here is not zero, we may express $R_{b c}$ in terms of $g_{b c} R$ and $g_{b c}$, and consequently modify $B$ at pleasure in (54), provided we make corresponding changes in $C$ and $D$. Thus we may assume in all cases

$$
A+(2-n) B=0 .
$$

Similarly, if the second coefficient is not zero, we may, by a second.contraction, express $R$ as a scalar, and hence change $C$ in (49) by making the proper changes in $D$, so as to get in all cases

$$
-B+(1-n) C=0 .
$$


Finally, when these changes have been made, if necessary, (55) gives by contraction

$$
D(1-n)=0
$$

Solving (56), (57) and (58) for the constants in terms of $A$, and then dividing out by $A$, which is not zero, we reduce (54) to the form

$$
\begin{aligned}
R_{a b c d}+\frac{1}{n-2}\left(g_{a c} R_{b d}\right. & \left.+g_{b d} R_{a c}-g_{a d} R_{b c}-g_{b c} R_{a d}\right) \\
& -\frac{1}{(n-2)(n-1)}\left(g_{a c} g_{b d}-g_{a d} g_{b c}\right) R=0 .
\end{aligned}
$$

In case any of the transformations of constants were necessary to make the coefficients of (55) vanish, (54) implies, in addition to (59), equations of type (44) and (47), but nothing further in any case.

Before considering the possibilities of combining these three types of equation, we shall prove that an equation of type (47) always leads to a special case of one of type (44), that in which the right member is a constant.* If (47) holds, we have

$$
R_{a / b}^{b}-\frac{1}{n}\left(R g_{a}^{b}\right)_{/ b}=0,
$$

denoting covariant derivatives in the usual way. On the other hand, it is well known, and easily proved by calculation in geodesic coordinates, that

$$
R_{a / b}^{b}-\frac{1}{2}\left(R g_{a}^{b}\right)_{/ b}=0
$$

identically, which shows that, unless $n=2$,

$$
\left(R g_{a}^{b}\right)_{/ b}=\partial R / \partial x_{a}=0, \quad R=R_{0} .
$$

When $n=2,(47)$ holds identically, so that from now on we need only consider the combination of (62) and (47).

In view of the preceding results, we see that all sets of equations of the kind discussed in this section are equivalent to one of the following five tensor equations:

* G. Herglotz, Leipziger Berichte, vol. 68 (1916), p. 203; cf. also G. D. Birkhoff. loc. cit., p. 220. 
(I) $R=M$,

(II) $R_{a b}-\frac{1}{n} g_{a b} R_{0}=0$,

(III) $R_{a b c d}+\frac{1}{n-2}\left(g_{a c} R_{b d}+g_{b d} R_{a c}-g_{a d} R_{b c}-g_{b c} R_{a d}\right)$

$$
\begin{array}{r}
-\frac{1}{(n-2)(n-1)}\left(g_{a c} g_{b d}-g_{a d} g_{b c}\right) R=0 \\
(\mathrm{IV})=\text { (III), (II) } R_{a b c d}+\frac{1}{n(n-1)}\left(g_{a c} g_{b d}-g_{a d} g_{b c}\right) R_{0}=0 \\
(\mathrm{~V})=\text { (III), (I) } R_{a b c d}+\frac{1}{n-2}\left(g_{a c} R_{b d}+g_{b d} R_{a c}-g_{a d} R_{b c}-g_{b c} R_{a d}\right) \\
-\frac{1}{(n-2)(n-1)}\left(g_{a c} g_{b d}-g_{a d} g_{b c}\right) M=0 .
\end{array}
$$

Here, as indicated, (IV) is equivalent to (III) and (II); while (V) is equivalent to (III) and (I). It is evident that (IV) follows from (III) and (II), and to deduce these from (IV) we have merely to contract (IV), obtaining (II), which then enables us to reduce (IV) to (III). We may handle (V) similarly. Since we have shown that (II) always implies the special case of (I)

$$
R=R_{0}
$$

these are all the combinations that we need consider. It should be particularly observed in the above that $R_{0}$ is a numerical constant, while $M$ is a scalar point function. Thus there is always some value of $M$ for which (I) holds - it only becomes a condition when the form of $M$ is given.

The equations above given may be interpreted geometrically. (I) states the value of the total curvature, and evidently only derives special significance when $M$ is specialized. (II) is the condition that the "principal directions" become indeterminate at every point of the space.* (III) is a necessary and sufficient condition $(n>3)$ that the space be conformally representable on

${ }^{*}$ L. P. Eisenhart, Proceedings of the National Academy of Sciences, vol. 8 (1922), p. 24. 
a euclidean space.* Its left member occurs in various investigations on conformal representation, and has been called the "conform curvature". (IV) is the condition that the space be "spherical".t

As stated previously, the equations holding in space time which are the analogues of Laplace's equation in the Newtonian theory of gravitation for the theory of Einstein must be of the type under discussion. Thus they must be one of the equations given in (63). Since (I) is not restrictive enough, and (III) is too restrictive, it follows that the only possible equation is (II), that selected by Einstein.

Recapitulating the work of this section, we have proved

THEOREM III. Every set of invariant equations formed by equating to zero expressions involving the fundamental quadratic tensor; $g_{i j}$, its first and second derivatives, and these last linearly, is equivalent to a tensor equation of one of the five types given in (63) above.

*H. Weyl, Mathematische Zeitschrift, vol. 2 (1918), p. 404; J. A. Schouten, Mathematische Zeitschrift, vol. 11 (1921), pp. 58ff.; cf. also H. W. Brinkmann, Proceedings of the National Academy of Sciences, vol. 9 (1923), p. 1, p. 172.

† Schouten, loc. cit., p. 75.

HARVARD UNIVERSITY, Cambridge, Mass. 